\title{
Exercise therapy for functional capacity in chronic diseases: an overview of meta-analyses of randomised controlled trials
}

\author{
Tero Pasanen, Samppa Tolvanen, Ari Heinonen, Urho M Kujala
}

- Additional material is published online only. To view please visit the journal online (http://dx.doi.org/10.1136/ bjsports-2016-097132).

Faculty of Sport and Health Sciences, University of Jyväskylä, Jyväskylä, Finland

Correspondence to Professor Urho M Kujala, Faculty of Sport and Health Sciences, University of Jyväskylä, Rautpohjankatu 8, P.0. Box 35, Jyväskylä, Finland FI-40014; Urho.M.Kujala@jyu.fi

Accepted 30 March 2017 Published Online First 12 May 2017

\section{SLinked}

http://dx.doi.org/10.1136/ bjsports-2017-097621

CrossMark

To cite: Pasanen T,

Tolvanen S, Heinonen A,

et al. Br J Sports Med

2017:51:1459-1465.
ABSTRACT

Objective To summarise all meta-analyses of randomised controlled trials that have evaluated the effects of exercise therapy on functional capacity in patients with chronic diseases.

Design Umbrella review of meta-analyses of randomised controlled trials.

Data sources We systematically searched the CENTRAL, CINAHL, DARE, Medline, OTSeeker, PEDro, SPORTDiscus, ProQuest Nursing \& Allied Health Database, Web of Science, Scopus, OpenGrey and BMC Proceedings from database inception to 1 September 2016.

Eligibility criteria for selecting studies We included meta-analyses that compared the effects of exercise therapy with no treatment or usual care in adults with non-communicable chronic diseases and included outcomes related to functional capacity. We excluded meta-analyses with less than 100 patients.

Results Eighty-five meta-analyses with 22 different chronic diseases were included. The exercise interventions resulted in statistically significant $(p<0.05)$ improvements for 126 of 146 (86\%) functional capacity outcomes, compared with the control group. The standardised mean differences were small in 64 (44\%), moderate in $54(37 \%)$ and large in $28(19 \%)$ of the 146 functional capacity outcomes. The results were similar for aerobic exercise, resistance training, and aerobic and resistance training combined. There were no significant differences in serious adverse effects between the intervention and control groups in any of the metaanalyses.

Conclusion Exercise therapy appears to be a safe way to improve functional capacity and reduce disability in individuals with chronic disease.

\section{INTRODUCTION}

Chronic diseases place high economic burden on society and reduce the quality of life of more than a billion individuals that live with a disability. ${ }^{1-4}$ Furthermore, almost half of the global population suffers from at least one chronic disease and may, therefore, be at risk for reduced functional capacity and disability. ${ }^{56}$ Disability is defined by WHO in the International Classification of Functioning, Disability and Health (ICF) as 'an umbrella term for impairments, activity limitations and participation restrictions'. ${ }^{7}$ Traditionally, medical research on physical activity or exercise has focused on the effects of exercise on disease risk factors, prevention, possible disease progression and risk of death. However, physical functioning is important for the activities of daily living of the patient and there is a need for more resources for overall patient care, particularly among the increasing number of older people.

During past decades, exercise training has been increasingly used in the treatment of chronic diseases and specialists have started to use the terms 'exercise therapy' and 'therapeutic exercise'. Exercise therapy for chronic diseases can be broadly categorised as aerobic, resistance, combination of aerobic and resistance, or other condition-specific exercise-based training. Condition-specific exercise is used to target specific functional impairments; for instance, supervised walking training or balance training may be used to improve walking ability in patients with stroke. To measure functional capacity, either objective measures of performance, such as maximal oxygen uptake $\left(\mathrm{VO}_{2 \max }\right)$, or patient-reported measures, such as the SF-36 Physical Function Scale, may be used. ${ }^{8}$ Disability is evaluated using patient-reported measures and is determined as the degree of loss in functional capacity. ${ }^{4}$

Several meta-analyses have found exercise therapy improves functional capacity and reduces disability in individuals with chronic diseases, ${ }^{9}{ }^{10}$ but due to the large number of randomised controlled trials (RCTs) and meta-analyses of RCTs, an umbrella summary of the results is necessary. Knowing in which conditions physical training should be added to therapy protocols is important for the patient and for the clinician's decision making. Therefore, the purpose of this systematic review was to conduct an umbrella review ${ }^{11}$ of all meta-analyses of RCTs that have evaluated the effects of exercise therapy on the physical performance, functional capacity, or disabilities of patients with chronic non-communicable diseases when compared with usual care. This systematic review design has been planned on the basis of previous reviews of the multidimensional effects of exercise therapy in different chronic diseases. ${ }^{12} 13$ This evidence-based knowledge is important for professionals in sports and exercise medicine and physiotherapy and for specialists in other fields to understand the important role of exercise therapy in healthcare.

\section{METHODS}

This updated review, based on the overview of reviews protocol by the Cochrane Collaboration, ${ }^{14}$ focuses solely on the physical performance and functional capacity outcomes, which are theoretically the most direct and quickly achieved benefits of exercise therapy. 


\section{Search strategy and selection criteria}

A systematic search was conducted using the following bibliographic databases: CENTRAL (via OvidSP), CINAHL (via EBSCO), DARE (via OvidSP), Medline (via PubMed), OTSeeker, PEDro, SPORTDiscus (via EBSCO), ProQuest Nursing \& Allied Health Database, Web of Science, Scopus, OpenGrey and BMC Proceedings from their inception to 1 September 2016. To retrieve additional articles, Google Scholar was used to search for relevant clinical guidelines and systematic reviews for more references, the reference lists of the included meta-analyses were screened manually and the personal archives of the authors were searched. Article language and publication status were not restricted. The search phrase consisted of MeSH terms and keywords related to exercise, meta-analysis and chronic disease (see online supplementary table 1).

Eligible articles were required to (1) be meta-analyses of RCTs, (2) have outcome measures related to physical performance, functional capacity or disability, (3) compare exercise therapy with no treatment or usual care, (4) have mostly adult participants, as defined by the Cochrane Collaboration ${ }^{14}$ and (5) have patients with any non-communicable chronic disease as defined by WHO. ${ }^{15}$ Meta-analyses were excluded if (1) they did not report the number of studies or participants, (2) they assessed postsurgical recovery or site-specific musculoskeletal conditions such as patellofemoral pain syndrome (although exercise therapy may be a standard treatment), (3) the exercise intervention was of very low intensity, for example, yoga, or (4) the meta-analysis had the same outcome as another meta-analysis assessing the same disease and intervention that was newer, higher quality as assessed with AMSTAR checklist ${ }^{16}$ or with a higher sample size.

\section{Data analysis}

Article screening, data extraction and quality assessment were carried out by two authors independently (TP, ST). Discrepancies were resolved by discussion or by consulting the senior authors (AH, UMK). First, the titles and abstracts were screened, then the full-text articles were retrieved and evaluated against the inclusion criteria. Articles that were not available were requested from the authors. Then, outcome data at the end of the intervention, intervention characteristics, publication bias assessments and adverse effects were extracted. Finally, the quality of each included meta-analysis was evaluated using the AMSTAR checklist. ${ }^{16}$ For results that were not presented as standardised mean difference (SMD) in the original meta-analysis, the Cochrane Collaboration's Review Manager V.5.3 was used to convert the outcomes to SMD to allow visual comparison of the results in a forest plot. If data were insufficient for conversion, data were extracted from original RCTs and processed as instructed by the Cochrane Collaboration. ${ }^{14}$

The meta-analyses were classified according to the type of exercise therapy into four categories:

1. Aerobic exercise

2. Resistance training

3. Aerobic and resistance training combined

\section{Other condition-specific exercise-based training}

For each exercise category, the data were analysed qualitatively based on the SMDs of each included outcome. The SMDs were evaluated using forest plot figures as well as proportions of significant results and proportions of small (SMD <0.5), moderate (SMD 0.5-0.8) and large (SMD >0.8) effect sizes. ${ }^{17}$ The significance of the results $(p<0.05)$ was judged based on the $95 \%$ CIs of the SMDs. In addition to this primary analysis, the results were evaluated qualitatively based on the mean differences (MDs). We did not include meta-analyses with less than three studies or less than 100 participants in the main analysis. We tested if these meta-analyses would have changed the results by performing a qualitative sensitivity analysis by adding the results of these meta-analyses in the figures and comparing the results visually.

\section{RESULTS}

The search process is presented in a flow diagram in figure 1 . The systematic search retrieved a total of 11947 articles and seven additional records were identified through the manual search. After removing duplicates, 10425 articles remained; of these, 10108 articles were excluded based on their titles and abstracts. The remaining 317 articles were retrieved as full-text versions. Of these articles, 233 were excluded (see online supplementary table 2) and finally, 84 meta-analyses were included in the qualitative synthesis. Of these, 13 meta-analyses were excluded from the report figures (see online supplementary table 3). Our search strategy resulted in the inclusion of meta-analyses with the following chronic diseases: Alzheimer's disease, cancer, chronic fatigue syndrome, chronic heart failure, chronic kidney disease, chronic obstructive pulmonary disease, cognitive impairment, coronary heart disease, dementia, fibromyalgia, haematological malignancies, heart failure, interstitial lung disease, multiple sclerosis, osteoarthritis, Parkinson's disease, peripheral arterial disease (intermittent claudication), rheumatoid arthritis, stroke and type 2 diabetes.

\section{Meta-analysis quality}

The AMSTAR Score ranged from 1 to 11 out of 11 with a median of 6 which indicates moderate overall methodological quality (see online supplementary table 4 ). A quarter (21 of the 85 included meta-analyses) assessed publication bias. Eleven of $147(7 \%)$ outcomes were reported to have publication bias, although it was minimal in almost all cases. The outcomes with potential publication bias are indicated in figures $2-5$.

\section{Aerobic exercise interventions}

Thirteen meta-analyses with aerobic exercise interventions, ${ }^{18-30}$ were included in the qualitative synthesis, and two eligible meta-analyses ${ }^{31} 32$ were excluded from the figures as they were older, lower quality or had a lower sample size than other meta-analyses with the same disease and outcome. Participant characteristics for the meta-analyses are presented in online supplementary table 5 . The meta-analyses included land-based exercise only, water-based exercise only or both. Land-based exercise included walking, cycling, circuit training, and sports such as basketball and soccer. There was considerable heterogeneity in the interventions included in the meta-analyses. The length of interventions ranged from 2 weeks to 104 weeks. The exercise frequency was 1-10 times per week, the duration was $10-180 \mathrm{~min}$ per session, and the intensity was $27 \%-95 \%$ of maximum heart rate (for detailed intervention characteristics, see online supplementary tables 6-10). The exercise interventions resulted in statistically significant improvements for 28 of $31(90 \%)$ outcomes, compared with the control group (see figure 2). The SMDs were small, moderate and large in $45 \%$, $45 \%$ and $10 \%$ of the outcomes, respectively. For results as MD, see online supplementary table 11 . Results were similar regardless of whether objective or patient self-reported measures of functional capacity were used. 
11947 records identified through database searching
7 additional records identified through other sources

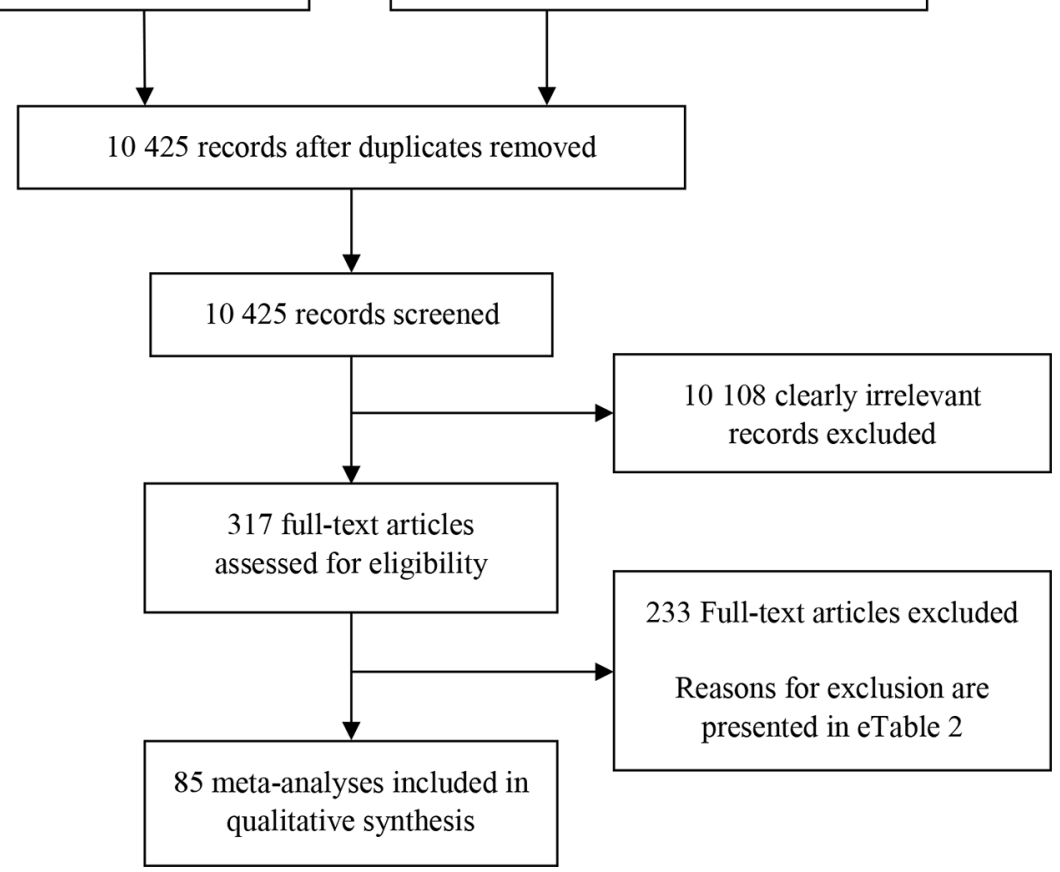

Figure 1 Flow diagram of the study selection process.

\section{Resistance training interventions}

Eleven meta-analyses with resistance training interventions, ${ }^{30} 33-42$ were included in the qualitative synthesis and two eligible meta-analyses ${ }^{43}$ were excluded from the figures. The length of the interventions ranged from 2 weeks to 104 weeks. The exercise frequency was two to seven times per week, the duration was 6-90 min per session, and the volume and repetition range were one to four sets per exercise and 5 to 30 repetitions per set, respectively. The equipment used varied from traditional free weights to therapeutic tools, such as an isokinetic dynamometer. The exercise interventions resulted in statistically significant improvements for 17 of 19 (89\%) outcomes, compared with the control group (see figure 3 ). The SMDs were small, moderate and large in $42 \%, 37 \%$ and $21 \%$ of the outcomes, respectively.

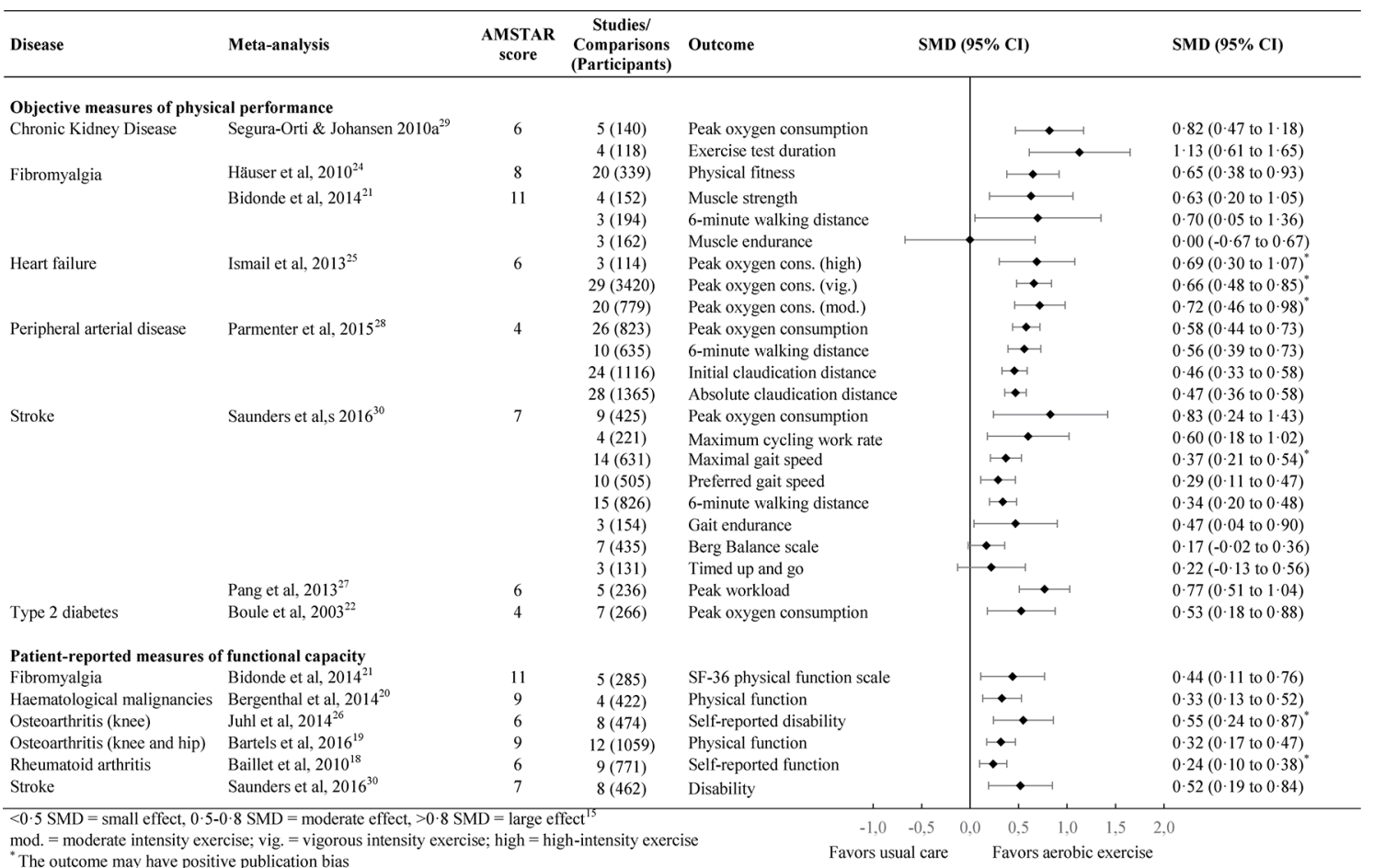

Figure 2 Effect of aerobic exercise on functional capacity in chronic diseases. SMD, standardised mean difference. 


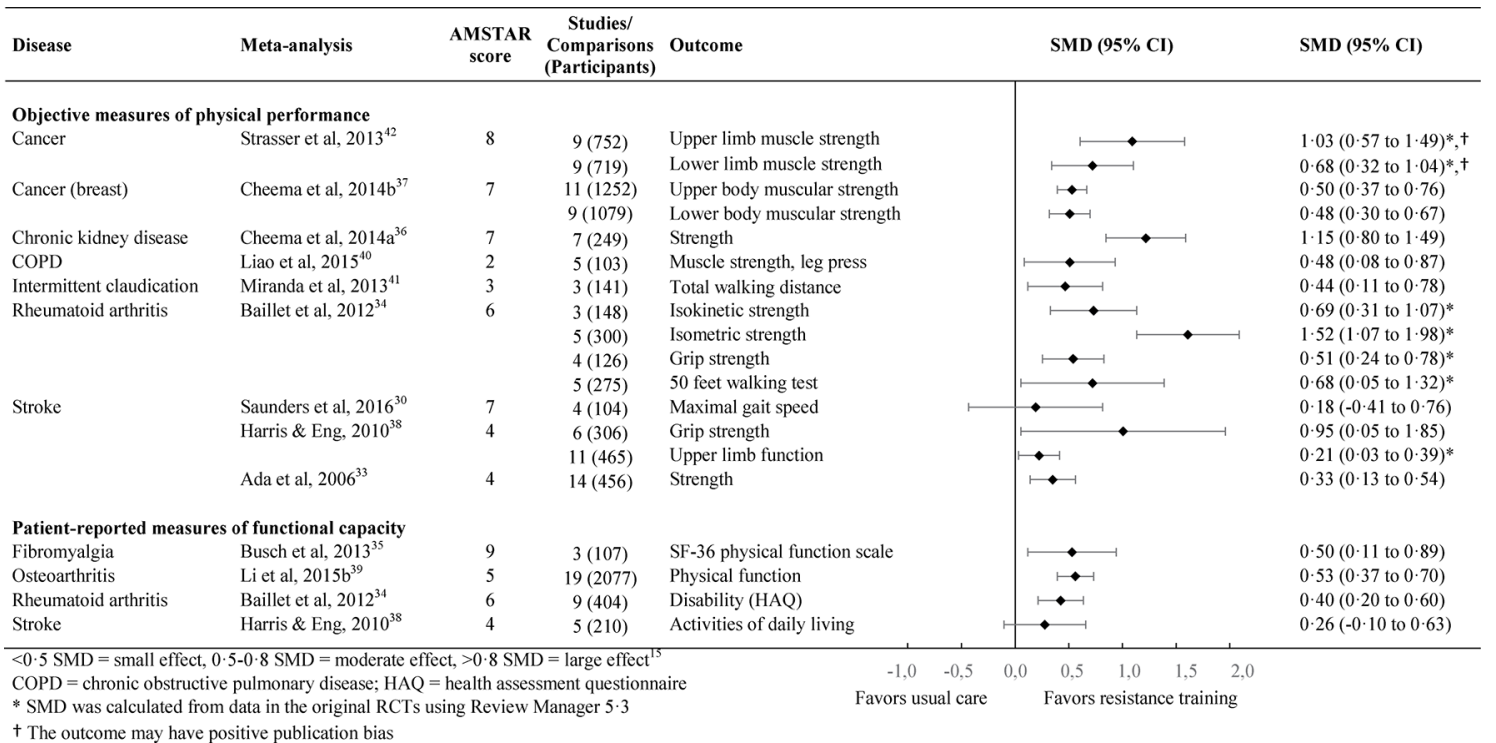

Figure 3 Effect of resistance training on functional capacity in chronic diseases. SMD, standardised mean difference.

\section{Combined aerobic and resistance training interventions}

Thirty-two meta-analyses with combined aerobic and resistance training interventions ${ }^{30} 45-75$ were included in the qualitative synthesis and nine eligible meta-analyses ${ }^{28}{ }^{76-83}$ were excluded from the figures. The length of the interventions ranged from 2 weeks to 112 weeks. The characteristics of interventions were similar to those in the separate aerobic or resistance training interventions. The exercise interventions resulted in statistically significant improvements for 54 of 60 (90\%) outcomes, compared with the control group (see figure 4). The SMDs were small, moderate and large in $40 \%, 42 \%$ and $18 \%$ of the outcomes, respectively.

\section{Condition-specific exercise}

Twenty meta-analyses with condition-specific interventions ${ }^{26} 41$ 84-101 were included in the qualitative synthesis and two meta-analyses ${ }^{102} 103$ were excluded from the figures. Condition-specific exercise was conducted both individually and in groups. The interventions included, for example, supervised walking therapy and specific physical rehabilitation approaches such as dual-task walking, transfer practice, specific aerobic exercises and resistance training. In most studies, the exercise was supervised by physiotherapists or occupational therapists. The length of interventions ranged from 2 weeks to 104 weeks. The exercise frequency was 1-14 times per week for 10-300 min per session. In the few meta-analyses that reported exercise intensity, it was low to moderate. The exercise interventions resulted in statistically significant improvements for 27 of 36 (75\%) outcomes (see figure 5). The SMDs were small, moderate and large in $47 \%, 25 \%$ and $28 \%$ of the outcomes, respectively.

\section{Sensitivity analysis}

To test if meta-analyses with less than three studies or less than 100 participants would have changed the results of this study we added the results of these meta-analyses in the figures to see if they changed the results. The results appeared to be in agreement with larger meta-analyses (the mean indicated a low to moderate positive effect), however, due to the very wide CIs, the results could not be interpreted reliably. That is, the often non-significant effects were probably not caused by inefficiency of exercise therapy but rather by the small sample size.

\section{Safety}

None of the meta-analyses reported a statistically significant difference in adverse effects, deaths or hospitalisations between the exercise and control groups. Information about the adverse effects was available in 64 of $85(75 \%)$ meta-analyses; although, most of the meta-analyses reported insufficient reporting of adverse effects in the original RCTs. The adverse effects experienced by control and intervention group participants ranged from increased pain to myocardial infarction (see online supplementary table 12).

\section{DISCUSSION}

The main finding of this umbrella review was that exercise therapy can improve physical performance and functional capacity in all of the included non-communicable chronic diseases. In approximately half of the physical performance and functional capacity outcomes, the effect of exercise was moderate or large. Therefore, the improvements are likely to be clinically important through reduction of functional capacity limitations in everyday activities. Although adverse effects were not consistently reported, based on the available data, exercise therapy appears to be safe in all of the included chronic diseases. The results appear to be similar for all training types, regardless of whether the studies used objective or patient-reported outcomes. However, condition-specific programmes had a lower proportion of significant results compared with aerobic exercise, strength training and combined aerobic and strength training. The finding that physical performance and functional capacity are improved by exercise therapy, is in agreement with the meta-analyses that were excluded from the qualitative synthesis as well as meta-analyses where multiple chronic diseases had been combined. ${ }^{910}$

\section{Validity of the results}

At the time of writing this overview, there were 13 meta-analysis protocols that would have fulfilled the inclusion criteria for this overview in the PROSPERO International Prospective Register of Systematic Reviews. It appears unlikely that future meta-analyses would change the overall conclusion of this overview, however, future research will likely increase our disease-specific knowledge on many specific aspects of exercise therapy. It is 


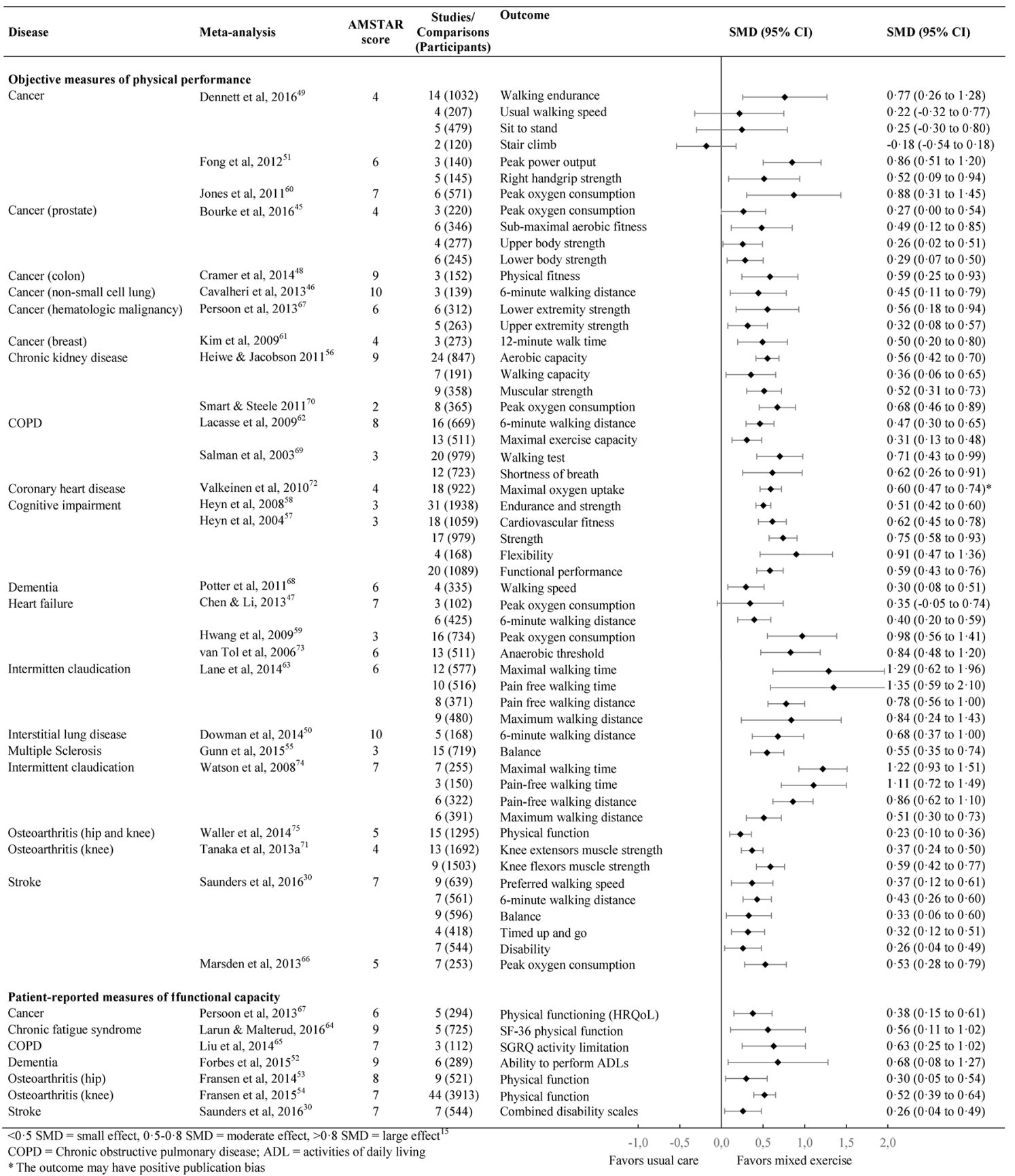

Figure 4 Effect of missed exercise on functional capacity in chronic diseases. SMD, standardised mean difference.

also unlikely that expanding this overview to include systematic reviews without meta-analysis would change the results considerably as the vast majority of systematic reviews appear to be in agreement with these results. ${ }^{104}$

\section{Generalisability of the results}

The results of this review are applicable to adult patients with any one of the included chronic diseases. As it is possible that meta-analyses have not been conducted or only a few RCTs are available for some chronic diseases for which exercise therapy might not be effective, our results cannot be directly generalised to chronic diseases that were not included in this review. Our review covers most non-communicable chronic diseases with high prevalence in the general population. For cancer, the results can be generalised mostly to cancer survivors after aggressive cancer treatments.

\section{Impact of exercise therapy}

Perhaps one of the most important impacts of improved functional capacity is improved coping with activities of daily living. This may also contribute to reduced experience of pain and disease progression, as well as psychological benefits. ${ }^{105}$ Improved functional capacity of elderly patients has important economic benefits due to reduced need of care and the ability to live at home. The results of this study are in line with a recent RCT showing that a structured physical activity programme reduced mobility disability among older, at-risk adults. ${ }^{106}$ Increasing functional capacity and functional reserve 


\begin{tabular}{|c|c|c|c|c|c|c|c|}
\hline Disease & Meta-analysis & $\begin{array}{c}\text { AMSTAR } \\
\text { score }\end{array}$ & $\begin{array}{c}\text { Studies/ } \\
\text { Comparisons } \\
\text { (Participants) }\end{array}$ & Outcome & & SMD $(95 \% \mathrm{CI})$ & SMD $(95 \% \mathrm{CI})$ \\
\hline \multicolumn{8}{|c|}{ Objective measures of physical performance } \\
\hline Alzheimer's disease & Rao et al, $2014^{95}$ & 2 & $6(446)$ & Physical function & & $\longmapsto$ & $0.53(0.24$ to 0.82$)$ \\
\hline Chronic heart failure & Gomes Neto et al, 2014a ${ }^{87}$ & 4 & $2(123)$ & Peak oxygen consumption & & & $4.86(2.81$ to 6.91$)$ \\
\hline \multirow[t]{3}{*}{ COPD } & McCarthy et al, $2015^{89}$ & 8 & $8(694)$ & Maximal Exercise & & $\longmapsto$ & $0.44(0.15$ to 0.74$)$ \\
\hline & & & $16(779)$ & Maximal Exercise Capacity & & $\longmapsto$ & $0.33(0.13$ to 0.53$)$ \\
\hline & & & 38 (1879) & 6-minute walking distance & & $\longmapsto$ & $0.63(0.46$ to 0.80$)$ \\
\hline \multirow[t]{3}{*}{ Intermittent claudication } & Miranda et al, $2013^{41}$ & 3 & $5(284)$ & Claudication distance & & $\longmapsto$ & $1.07(0.81$ to 1.34$)$ \\
\hline & Fakhry et al, $2012^{86}$ & 5 & $24(916)$ & Maximum walking distance & & $\longmapsto$ & $1.31(0.86 \text { to } 1.76)^{*}$ \\
\hline & & & $20(708)$ & Pain-free walking distance & & $\longmapsto$ & $1.27(0.81 \text { to } 1.73)^{*}$ \\
\hline Osteoarthritis & Wallis \& Taylor $2011^{99}$ & 5 & $4(240)$ & Gait performance & $\longmapsto$ & $\bullet$ & $0 \cdot 12(-0 \cdot 30$ to $0 \cdot 55)$ \\
\hline \multirow[t]{6}{*}{ Parkinson Disease } & Shen et al, $2016^{96}$ & 3 & $24(1881)$ & Postural stability & & $\mapsto-1$ & $0.30(0.21$ to 0.40$)$ \\
\hline & Tomlinson et al, $2013^{98}$ & 9 & $3(145)$ & $10 \mathrm{~m}$ or $20 \mathrm{~m}$ walk test & $\longmapsto$ & $\longrightarrow$ & $-0 \cdot 10(-0 \cdot 43$ to $0 \cdot 23)$ \\
\hline & & & $5(248)$ & Gait speed & $\mapsto$ & 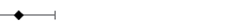 & $0 \cdot 15(-0 \cdot 11$ to $0 \cdot 40)$ \\
\hline & & & $6(370)$ & Timed up and go & H & $\longrightarrow$ & $0 \cdot 13(-0.08$ to 0.33$)$ \\
\hline & & & $3(280)$ & Berg balance scale & & $\longrightarrow$ & $0.24(0.00$ to 0.47$)$ \\
\hline & Mehrholz et al, $2010^{90}$ & 10 & $7(153)$ & Gait speed & & $\longmapsto$ & $0.50(0.17$ to 0.84$)$ \\
\hline \multirow{3}{*}{ Peripheral arterial disease } & Lyu et al, $2016^{88}$ & 5 & $16(1119)$ & Maximum walking distance & & . & $1.15(0.75$ to 1.54$)$ \\
\hline & & & $11(550)$ & Pain free walking distance & & & $1.52(0.85$ to 2.18$)$ \\
\hline & & & $6(524)$ & 6-minute walking distance & & $\longmapsto$ & $0.45(0.27$ to 0.63$)$ \\
\hline \multirow[t]{13}{*}{ Stroke } & Etoom et al, $2016^{85}$ & 5 & $36(1473)$ & Upper extremity function & & $\longmapsto$ & $0.56(0.30$ to 0.81$)$ \\
\hline & Peurala et al, 2014 $4^{91}$ & 5 & $7(236)$ & Walking speed & & $\cdot$ & $0.57(0.03$ to 1.12$)$ \\
\hline & & & $6(211)$ & 6-minute walking distance & & $\longmapsto$ & $0.64(0.25$ to 1.02$)$ \\
\hline & Pollock et al, $2014 \mathrm{a}^{93}$ & 9 & $3(292)$ & Gait velocity & $\bullet$ & $\bullet$ & $0.05(-0.18$ to 0.28$)$ \\
\hline & & & $5(246)$ & Balance & & $\longmapsto$ & $0.31(0.05$ to 0.56$)$ \\
\hline & & & $27(4558)$ & Motor function & & $\longmapsto$ & $0.81(0.58$ to 1.04$)$ \\
\hline & Pollock et al, $2014 \mathrm{~b}^{94}$ & 8 & $7(335)$ & Ability to sit-to-stand & & $\longmapsto$ & $0.34(0.06$ to 0.62$)$ \\
\hline & & & $6(105)$ & Lateral symmetry & & $\longmapsto$ & $0.85(0.38$ to 1.33$)$ \\
\hline & Polese et al, $2013^{92}$ & 5 & $7(275)$ & $10 \mathrm{~m}$ walk test & & $\longmapsto \bullet$ & $0.69(0.45$ to 0.94$)$ \\
\hline & & & $6(249)$ & 6-minute walking distance & & $\longmapsto$ & $0.71(0.45$ to 0.97$)$ \\
\hline & States et al, $2009^{97}$ & 10 & $7(396)$ & Gait speed & & $\longmapsto$ & $0.30(0 \cdot 10$ to 0.50$)$ \\
\hline & & & $3(118)$ & Timed up and go & & $\longmapsto$ & $0.80(0.41$ to 1.20$)$ \\
\hline & & & $4(181)$ & 6-minute walking distance & & $\longmapsto$ & $0.35(0.05$ to 0.64$)$ \\
\hline \multicolumn{8}{|c|}{ Patient-reported measures of functional capacity } \\
\hline Alzheimer's disease & Rao et al, $2014^{95}$ & 2 & $6(446)$ & ADL performance & & $\longmapsto$ & $0.80(0.42$ to $1 \cdot 19)$ \\
\hline \multirow[t]{6}{*}{ Stroke } & Zhang et al, $2014^{101}$ & 5 & $31(5220)$ & Barthel Index & & $\longmapsto$ & $1.04(0.88$ to 1.21$)$ \\
\hline & & & $27(4501)$ & Impairment & & $\longmapsto$ & $1 \cdot 10(0.82$ to 1.38$)$ \\
\hline & Pollock et al, $2014 \mathrm{a}^{93}$ & 9 & $27(3423)$ & Independence in ADLs & & $\longmapsto$ & $0.78(0.58$ to 0.97$)$ \\
\hline & Coupar et al, $2010^{84}$ & 9 & $3(106)$ & Performance in ADLs & $\vdash$ & $\longrightarrow$ & $0.25(-0.14$ to 0.63$)$ \\
\hline & States et al, $2009^{97}$ & 10 & $3(269)$ & Gait function & & $\longrightarrow$ & $0.19(-0.05$ to 0.43$)$ \\
\hline & & & $3(269)$ & Barthel Index & $\longmapsto$ & & $0.03(-0.21$ to 0.27$)$ \\
\hline \multicolumn{2}{|c|}{$\begin{array}{l}<0.5 \mathrm{SMD}=\text { small effect, } 0.5-0.8 \mathrm{SMD}=\text { moderat } \\
\text { * The outcome may have positive publication bias } \\
\mathrm{ADL}=\text { activities of daily living }\end{array}$} & & ect" & & 0,0 & 0,5 & \\
\hline
\end{tabular}

Figure 5 Effect of other condition-specific exercise-based rehabilitation modes (than those included in figures 2-4) on functional capacity in chronic diseases. SMD, standardised mean difference.

are important components of maintaining unrestricted mobility, independence and a high quality of life; therefore, paying attention to the patients' functional capacity should be a routine part of each medical examination.

\section{Disease-specific considerations}

Due to the scope of this overview we have not focused in detail on disease-specific results. Furthermore, detailed comparisons on how well individuals with different chronic diseases respond to exercise based on this overview might not be reliable as the methods used in the original RCTs and meta-analyses vary considerably. The differences in the results for different chronic diseases could be caused by a multitude of confounding factors that we have not systematically appraised in this overview, such as the quality and duration of the exercise programme design, supervision, adherence and disease severity. However, it is important to keep in mind that different diseases cause different types of functional impairments and exercise programmes should be designed to target those impairments. Improving aerobic performance while influencing cardiometabolic risk factors is important in diseases such as type 2 diabetes and coronary heart disease; however, training specific muscle groups may be necessary when improving the functional capacity of patients with specific musculoskeletal problems. Our results concerning the types of training used in patients with different diseases reflect these disease-specific needs. Comparing the effect sizes of different types of exercise on different outcomes would not be valid using this study design, but different types of training programmes (aerobic, strength and both combined) appear to give the specific desirable benefits consistently. There was a good match between the objective measurements and self-reported functional benefits. The lower proportion of significant positive effects due to condition-specific exercise may be related to the more severe functional limitations among certain patient groups, such as those with Parkinson's disease or those with severe (preoperative) osteoarthritis. Moreover, usual care also included physical training components, for example, in the treatment of patients with stroke. So, the condition-specific programmes in our classification are often carried out in situations where the disease sets limitations to training and recovery.

\section{Implications for practice}

When applying the results of this umbrella review in clinical practice, consider that the patients usually did not have comorbidities in the original RCTs. In addition, in the scientific trials the inclusion and exclusion criteria are usually carefully planned and followed, and the training programmes are tailored and guided according to the disease type and severity, which may have reduced the number of complications. ${ }^{13}$ In clinical work disease-specific functional and mobility limitations should be considered and identified as early as possible, to prevent them from worsening. Also, the type of exercise therapy programme should be selected to best correct the disease-specific functional problems. The supplementary tables with the exercise intervention characteristics and the included meta-analyses may be used as a basis for designing exercise programmes for different chronic diseases. Usually the training programme should be progressive and include follow-ups to document the adherence, effectiveness 
and possible adverse effects. It is a challenge for the healthcare system and its professionals to address these issues in real clinical situations. A recent study reported that among adults with disabilities who had visited a health professional within the past 12 months, $56 \%$ of did not receive a recommendation for physical activity. ${ }^{107}$

\section{Recommendations for future research}

The development of reporting guidelines for adverse effects is warranted, as there appears to be a serious lack of consistency in reporting in the RCTs included in the meta-analyses. Moreover, very few meta-analyses fulfilled the 11th criteria in the AMSTAR checklist: 'was the conflict of interest included?' We also encourage development and inclusion of proper cost-benefit analyses in future exercise therapy studies, since the current trials usually do not provide adequate data for such analyses. These studies should include both disease-specific and general outcome measures and collaboration with cross-sector partners. Moreover, currently there seems to be great variation in the exercise programme designs and lengths of interventions, which indicates that meta-regression and network meta-analyses are needed to find out the most optimal ways to improve functional capacity in different chronic diseases. Finally, although we used WHO's ICF framework's definition of disability, the patient-reported outcome measures used in the included meta-analyses to describe disability may not have been developed using ICF as a guiding framework. Evaluating how these outcome measures fit with the ICF framework, could be another focus for future research.

\section{Strengths and limitations}

The main strength of our umbrella review was that we only included meta-analyses with RCTs. Moreover, the likelihood of retrieving all relevant articles was maximised by using a highly sensitive search strategy. However, there were some limitations to our study. First, we did not publish a protocol for this study. Nonetheless, to our knowledge, this would not have changed the way the study was conducted, although, it might have made us exclude very low intensity exercise protocols from the review earlier. Another limitation was low methodological quality in some meta-analyses. Nevertheless, the results of the lower quality meta-analyses seemed to be in line with those with higher AMSTAR scores. It is possible that many of the lower scores also reflect poor reporting standards rather than poor methodological quality. The effect of publication bias on the results remains unclear, as publication bias was statistically analysed for only a third of the outcomes. However, in almost all instances, it was reported to be minimal. Therefore, it likely does not have a large impact on the vast majority of results. The quality of the presented evidence is unclear as the GRADE quality evaluation ${ }^{108}$ was available for only approximately a tenth of the outcomes. For these outcomes, the ratings ranged from low to high. When including several meta-analyses, it is possible for a single study to be included in more than one meta-analysis. However, since we did not include overlapping meta-analyses on the same outcome and the same chronic disease, this was unlikely in this overview.

\section{Deviations from the unpublished protocol}

We included yoga and taiji in the search strategy as we had preplanned to include these interventions in the review. However, after conducting the search, we decided to exclude these forms of exercise with very low or unclear intensity as meta-analyses did not appear to distinguish the results between

\section{What is already known?}

- Worldwide chronic diseases reduce the functional capacity and quality of life of more than a billion individuals.

- Meta-analyses suggest that exercise therapy improves functional capacity and reduces disability in individuals with different chronic diseases, but due to the large number of randomised controlled trials (RCTs) and meta-analyses of RCTs, it is challenging for clinicians to summarise the results.

\section{What are the new findings?}

- Our umbrella review of meta-analyses shows that aerobic, resistance, and the combination of aerobic and resistance training have beneficial effects on objective and selfreported measures of functional capacity in patients with non-communicable chronic diseases.

- Exercise therapy appears to be safe for patients with non-communicable chronic diseases.

\section{How might it impact on clinical practice in the future?}

Exercise therapy should be recommended for all patients with non-communicable chronic diseases to prevent the decline of functional capacity and to improve it further.

less intensive and more intensive variants of these types of exercise. Without additional information regarding the intensity of exercise, interpretation of the results from the figures could have been challenging. Yoga and taiji may also have effects via other mechanisms than exercise itself.

\section{CONCLUSION}

Exercise therapy appears to be a safe way to improve physical performance and functional capacity, and reduce disability in individuals with non-communicable chronic disease. This applies to aerobic exercise, resistance training, combined aerobic and resistance training, and to most of the other condition-specific training protocols.

Contributors TP, AH and UMK created the study concept and design. TP, ST, $\mathrm{AH}$ and UMK carried out the acquisition, analysis and interpretation of data. TP drafted the manuscript. AH and UMK critically revised the manuscript for important intellectual content. TP conducted statistical analyses. UMK obtained funding. AH and UMK provided administrative, technical and material support. AH and UMK supervised the study.

Funding The study was supported by JYPE (collaborative research funding organisation of University of Jyväskylä and Rehabilitation Center Peurunka).

Competing interests TP is employed at Kuntokeskus Energy Oy as a physical therapist and administrative assistant. ST is employed at Kunnonpaikka as a physical therapist.

Provenance and peer review Not commissioned; externally peer reviewed.

(c) Article author(s) (or their employer(s) unless otherwise stated in the text of the article) 2017. All rights reserved. No commercial use is permitted unless otherwise expressly granted.

\section{REFERENCES}

1 Kohl HW, Craig CL, Lambert EV, et al. The pandemic of physical inactivity: global action for public health. Lancet 2012;380:294-305 
2 Parker L, Moran GM, Roberts LM, et al. The burden of common chronic disease on health-related quality of life in an elderly community-dwelling population in the UK. Fam Pract 2014;31:557-63.

3 Vos T, Flaxman AD, Naghavi M, et al. Years lived with disability (YLDs) for 1160 sequelae of 289 diseases and injuries 1990-2010: a systematic analysis for the global burden of disease study 2010. Lancet 2012;380:2163-96.

4 World Health Organization. World report on disability 2011. Geneva: World Health Organization, 2011.

5 Barnett K, Mercer SW, Norbury M, et al. Epidemiology of multimorbidity and implications for health care, research, and medical education: a cross-sectional study. Lancet 2012;380:37-43.

6 Violan C, Foguet-Boreu Q, Flores-Mateo G, et al. Prevalence, determinants and patterns of multimorbidity in primary care: a systematic review of observational studies. PLoS One 2014;9:e102149.

7 World Health Organization. Towards a common language for functioning, disability and health ICF. Geneva: World Health Organization, 2002.

8 Mannerkorpi K, Svantesson U, Broberg C. Relationships between performancebased tests and patients' ratings of activity limitations, self-efficacy, and pain in Fibromyalgia. Arch Phys Med Rehabil 2006;87:259-64.

9 Kelley GA, Kelley KS, Hootman JM, et al. Effects of community-deliverable exercise on pain and physical function in adults with arthritis and other rheumatic diseases: a meta-analysis. Arthritis Care Res 2011;63:79-93.

10 Desveaux L, Beauchamp M, Goldstein R, et al. Community-based exercise programs as a strategy to optimize function in chronic disease: a systematic review. Med Care 2014:52:216-26.

11 Ioannidis J. Next-generation systematic reviews: prospective meta-analysis, individual-level data, networks and umbrella reviews. Br J Sports Med 2017:bjsp orts-2017-097621 (Published Online First: 21 February 2017).

12 Kujala UM. Evidence for exercise therapy in the treatment of chronic disease based on at least three randomized controlled trials--summary of published systematic reviews. Scand I Med Sci Sports 2004;14:339-45.

13 Kujala UM. Evidence on the effects of exercise therapy in the treatment of chronic disease. Br J Sports Med 2009;43:550-5.

14 Higgins JPT, Green S. Cochrane handbook for systematic reviews of interventions version 5.1.0 [updated march 2011], 2011. www.handbook. cochrane.org.

15 World Health Organization. Global status report on noncommunicable diseases 2010. Geneva:World Health Organization, 2010. http://whqlibdoc.who.int/ publications/2011/9789240686458_eng.pdf.

16 Shea BJ, Hamel C, Wells GA, a WG, et al. AMSTAR is a reliable and valid measurement tool to assess the methodological quality of systematic reviews. J Clin Epidemiol 2009;62:1013-20.

17 Cohen J. Statistical power analysis for the behavioral sciences. London: Academic Press, 1988.

18 Baillet A, Zeboulon N, Gossec L, et al. Efficacy of cardiorespiratory aerobic exercise in rheumatoid arthritis: meta-analysis of randomized controlled trials. Arthritis Care Res 2010:62:984-92.

19 Bartels EM, Juhl CB, Christensen R, et al. Aquatic exercise for the treatment of knee and hip osteoarthritis. Cochrane Database Syst Rev 2016:3:CD005523.

20 Bergenthal N, Will A, Streckmann F, et al. Aerobic physical exercise for adult patients with haematological malignancies. Cochrane Database Syst Rev 2014;11:CD009075.

21 Bidonde J, Busch AJ, Webber SC, et al. Aquatic exercise training for Fibromyalgia. Cochrane Database Syst Rev 2014;10:CD011336.

22 Boulé NG, Kenny GP, Haddad E, et al. Meta-analysis of the effect of structured exercise training on cardiorespiratory fitness in type 2 diabetes mellitus. Diabetologia 2003;46:1071-81

23 Edmonds M, McGuire H, Price JR. Exercise therapy for chronic fatigue syndrome. Cochrane Database Syst Rev 2013;8:CD003200.

24 Häuser W, Klose P, Langhorst J, et al. Efficacy of different types of aerobic exercise in Fibromyalgia syndrome: a systematic review and meta-analysis of randomised controlled trials. Arthritis Res Ther 2010;12:R79.

25 Ismail H, McFarlane JR, Nojoumian AH, et al. Clinical outcomes and cardiovascular responses to different exercise training intensities in patients with heart failure: a systematic review and meta-analysis. JACC Heart Fail 2013;1:514-22.

26 Juhl C, Christensen R, Roos EM, et al. Impact of exercise type and dose on pain and disability in knee osteoarthritis: a systematic review and meta-regression analysis of randomized controlled trials. Arthritis Rheumatol 2014;66:622-36.

27 Pang MY, Charlesworth SA, Lau RW, et al. Using aerobic exercise to improve health outcomes and quality of life in stroke: evidence-based exercise prescription recommendations. Cerebrovasc Dis 2013;35:7-22.

28 Parmenter BJ, Dieberg G, Smart NA. Exercise training for management of peripheral arterial disease: a systematic review and meta-analysis. Sports Med 2015;45:231-44

29 Segura-Orti E, Johansen KL. Exercise in end-stage renal disease. Semin Dial 2010;23:422-30

30 Saunders DH, Sanderson M, Hayes S, et al. Physical fitness training for stroke patients. Cochrane Database Syst Rev 2016;3:CD003316.
31 Barker AL, Talevski J, Morello RT, et al. Effectiveness of aquatic exercise for musculoskeletal conditions: a meta-analysis. Arch Phys Med Rehabil 2014;95:1776-86.

32 Pang MY, Eng JJ, Dawson AS, et al. The use of aerobic exercise training in improving aerobic capacity in individuals with stroke: a meta-analysis. Clin Rehabil 2006:20:97-111.

33 Ada L, Dorsch S, Canning CG. Strengthening interventions increase strength and improve activity after stroke: a systematic review. Aust J Physiother 2006:52:241-8.

34 Baillet A, Vaillant M, Guinot M, et al. Efficacy of resistance exercises in rheumatoid arthritis: meta-analysis of randomized controlled trials. Rheumatology 2012:51:519-27.

35 Busch AJ, Webber SC, Richards RS, et al. Resistance exercise training for Fibromyalgia. Cochrane Database Syst Rev 2013;12:CD010884.

36 Cheema BS, Chan D, Fahey P, et al. Effect of progressive resistance training on measures of skeletal muscle hypertrophy, muscular strength and health-related quality of life in patients with chronic kidney disease: a systematic review and metaanalysis. Sports Med 2014:44:1125-38.

37 Cheema BS, Kilbreath SL, Fahey PP, et al. Safety and efficacy of progressive resistance training in breast Cancer: a systematic review and meta-analysis. Breast Cancer Res Treat 2014;148:249-68.

38 Harris JE, Eng JJ. Strength training improves upper-limb function in individuals with stroke: a meta-analysis. Stroke 2010;41:136-40

$39 \mathrm{Li}$ Y, Su Y, Chen S, et al. The effects of resistance exercise in patients with knee osteoarthritis: a systematic review and meta-analysis. Clin Rehabil 2016:30:947-59.

40 Liao WH, Chen JW, Chen X, et al. Impact of resistance training in subjects with COPD: a systematic review and Meta-Analysis. Respir Care 2015;60:1130-45.

41 Miranda A, Rodrigues LBdeCC, Rodrigues SLC, et al. Effects of walking and strength training on walking capacity in individuals with claudication: meta-analysis. J Vasc Bras 2013:12:110-7.

42 Strasser B, Steindorf K, Wiskemann J, et al. Impact of resistance training in Cancer survivors: a meta-analysis. Med Sci Sports Exerc 2013;45:2080-90.

43 Roddy E, Zhang W, Doherty M. Aerobic walking or strengthening exercise for osteoarthritis of the knee? A systematic review. Ann Rheum Dis 2005;64:544-8.

44 Mehta S, Pereira S, Viana R, et al. Resistance training for gait speed and total distance walked during the chronic stage of stroke: a meta-analysis. Top Stroke Rehabil 2012;19:471-8.

45 Bourke L, Smith D, Steed L, et al. Exercise for men with prostate Cancer: a systematic review and Meta-analysis. Eur Urol 2016;69:693-703.

46 Cavalheri V, Tahirah F, Nonoyama M, et al. Exercise training undertaken by people within 12 months of lung resection for non-small cell lung Cancer. Cochrane Database Syst Rev 2013;7:CD009955.

47 Chen YM, Li Y. Safety and efficacy of exercise training in elderly heart failure patients: a systematic review and meta-analysis. Int J Clin Pract 2013;67:1192-8.

48 Cramer $\mathrm{H}$, Lauche $\mathrm{R}$, Klose $\mathrm{P}$, et al. A systematic review and meta-analysis of exercise interventions for colorectal Cancer patients. Eur I Cancer Care 2014;23:3-14.

49 Dennett AM, Peiris CL, Shields N, et al. Moderate-intensity exercise reduces fatique and improves mobility in Cancer survivors: a systematic review and meta-regression. J Physiother 2016;62:68-82.

50 Dowman L, Hill C, Holland A. Pulmonary rehabilitation for interstitial lung disease. Cochrane Database Syst Rev 2014:6:CD006322.

51 Fong DY, Ho JW, Hui BP, et al. Physical activity for Cancer survivors: meta-analysis of randomised controlled trials. BMJ 2012;344:e70

52 Forbes D, Forbes SC, Blake CM, et al. Exercise programs for people with dementia. Cochrane Database Syst Rev 2015:4:CD006489.

53 Fransen M, McConnell S, Hernandez-Molina G, et al. Exercise for osteoarthritis of the hip. Cochrane Database Syst Rev 2014;4:CD007912.

54 Fransen M, McConnell S, Harmer AR, et al. Exercise for osteoarthritis of the knee. Cochrane Database Syst Rev 2015;1:CD004376

55 Gunn H, Markevics S, Haas B, et al. Systematic review: the effectiveness of interventions to reduce falls and improve balance in adults with multiple sclerosis. Arch Phys Med Rehabil 2015;96:1898-912.

56 Heiwe S, Jacobson SH. Exercise training for adults with chronic kidney disease. Cochrane Database Syst Rev 2011;10:CD003236.

57 Heyn P, Abreu BC, Ottenbacher KJ. The effects of exercise training on elderly persons with cognitive impairment and dementia: a meta-analysis. Arch Phys Med Rehabil 2004:85:1694-704.

58 Heyn PC, Johnson KE, Kramer AF. Endurance and strength training outcomes on cognitively impaired and cognitively intact older adults: a meta-analysis. J Nutr Health Aging 2008;12:401-9.

59 Hwang R, Marwick T. Efficacy of home-based exercise programmes for people with chronic heart failure: a meta-analysis. Eur I Cardiovasc Prev Rehabil 2009:16:527-35

60 Jones LW, Liang Y, Pituskin EN, et al. Effect of exercise training on peak oxygen consumption in patients with Cancer: a meta-analysis. Oncologist 2011;16:112-20.

61 Kim CJ, Kang DH, Park JW. A meta-analysis of aerobic exercise interventions for women with breast Cancer. West I Nurs Res 2009;31:437-61.

62 Lacasse Y, Goldstein R, Lasserson TJ, et al. Pulmonary rehabilitation for chronic obstructive pulmonary disease. Cochrane Database Syst Rev 2009;4:CD003793. 
63 Lane R, Ellis B, Watson L, et al. Exercise for intermittent claudication. Cochrane database Syst Rev 2014;7:CD000990.

64 Larun L, Brurberg KG, Odgaard-Jensen J, et al. Exercise therapy for chronic fatigue syndrome. Cochrane Database Syst Rev 2016;6:CD003200.

65 Liu XL, Tan JY, Wang T, et al. Effectiveness of home-based pulmonary rehabilitation for patients with chronic obstructive pulmonary disease: a meta-analysis of randomized controlled trials. Rehabil Nurs 2014;39:36-59.

66 Marsden DL, Dunn A, Callister R, et al. Characteristics of exercise training interventions to improve cardiorespiratory fitness after stroke: a systematic review with meta-analysis. Neurorehabil Neural Repair 2013;27:775-88.

67 Persoon S, Kersten MJ, van der Weiden K, et al. Effects of exercise in patients treated with stem cell transplantation for a hematologic malignancy: a systematic review and meta-analysis. Cancer Treat Rev 2013;39:682-90.

68 Potter R, Ellard D, Rees K, et al. A systematic review of the effects of physical activity on physical functioning, quality of life and depression in older people with dementia. Int I Geriatr Psychiatry 2011;26:1000-11.

69 Salman GF, Mosier MC, Beasley BW, et al. Rehabilitation for patients with chronic obstructive pulmonary disease: meta-analysis of randomized controlled trials. J Gen Intern Med 2003:18:213-21.

70 Smart N, Steele M. Exercise training in haemodialysis patients: a systematic review and meta-analysis. Nephrology 2011;16:626-32.

71 Tanaka R, Ozawa J, Kito N, et al. Evidence of improvement in various impairments by Exercise Interventions in patients with knee osteoarthritis: a systematic review and Meta-analysis of Randomized clinical trials. J Jpn Phys Ther Assoc 2013;16:7-21.

72 Valkeinen H, Aaltonen S, Kujala UM. Effects of exercise training on oxygen uptake in coronary heart disease: a systematic review and meta-analysis. Scand I Med Sc Sports 2010;20:545-55.

73 van Tol BA, Huiismans RJ, Kroon DW, et al. Effects of exercise training on cardiac performance, exercise capacity and quality of life in patients with heart failure: a meta-analysis. Eur J Heart Fail 2006:8:841-50.

74 Watson L, Ellis B, Leng GC. Exercise for intermittent claudication. Cochrane Database Syst Rev 2008;4:CD000990.

75 Waller B, Ogonowska-Slodownik A, Vitor M, et al. Effect of therapeutic aquatic exercise on symptoms and function associated with lower limb osteoarthritis: systematic review with meta-analysis. Phys Ther 2014;94:1383-95.

76 Busch AJ, Barber KA, Overend TJ, et al. Exercise for treating Fibromyalgia syndrome. Cochrane Database Syst Rev 2008;4:CD003786.

77 Chien CL, Lee CM, Wu YW, et al. Home-based exercise increases exercise capacity but not quality of life in people with chronic heart failure: a systematic review. Aust J Physiother 2008;54:87-93.

78 Escalante Y, García-Hermoso A, Saavedra JM. Effects of exercise on functional aerobic capacity in lower limb osteoarthritis: a systematic review. J Sci Med Sport 2011;14:190-8.

79 Jansen MJ, Viechtbauer W, Lenssen AF, et al. Strength training alone, exercise therapy alone, and exercise therapy with passive manual mobilisation each reduce pain and disability in people with knee osteoarthritis: a systematic review. J Physiother 2011;57:11-20.

80 Larun L, Malterud K. Treningsbehandling ved kronisk utmattelsessyndrom. Tidsskrift for Den norske legeforening 2011;131:231-6.

81 McNeely ML, Campbell KL, Rowe BH, et al. Effects of exercise on breast Cancer patients and survivors: a systematic review and meta-analysis. CMAJ 2006:175:34-41.

82 Rees K, Taylor RRS, Singh S, et al. Exercise based rehabilitation for heart failure. Cochrane Database Syst Rev 2004;3:CD003331.

83 Uthman OA, van der Windt DA, Jordan JL, et al. Exercise for lower limb osteoarthritis: systematic review incorporating trial sequential analysis and network meta-analysis. BMJ 2013;347:f5555-13.

84 Coupar F, Pollock A, Van Wijck F, et al. Simultaneous bilaternal training for improving arm function after stroke. Cochrane database Syst Rev Online 2010;4:CD006432.

85 Etoom M, Hawamdeh M, Hawamdeh Z, et al. Constraint-induced movement therapy as a rehabilitation intervention for upper extremity in stroke patients: systematic review and meta-analysis. Int J Rehabil Res 2016;39:197-210.
86 Fakhry F, van de Luijtgaarden KM, Bax L, et al. Supervised walking therapy in patients with intermittent claudication. J Vasc Surg 2012;56:1132-42.

87 Gomes Neto M, Menezes MA, Oliveira Carvalho V. Dance therapy in patients with chronic heart failure: a systematic review and a meta-analysis. Clin Rehabil 2014;28:1172-9

88 Lyu X, Li S, Peng S, et al. Intensive walking exercise for lower extremity peripheral arterial disease: a systematic review and meta-analysis. J Diabetes 2016;8:363-77

89 McCarthy B, Casey D, Devane D, et al. Pulmonary rehabilitation for chronic obstructive pulmonary disease. Cochrane database Syst Rev 2015;2:CD003793.

90 Mehrholz J, Friis R, Kugler J, et al. Treadmill training for patients with Parkinson's disease. Cochrane Database Syst Rev 2010;1:CD007830.

91 Peurala SH, Karttunen AH, Sjögren T, et al. Evidence for the effectiveness of walking training on walking and self-care after stroke: a systematic review and meta-analysis of randomized controlled trials. J Rehabil Med 2014;46:387-99.

92 Polese JC, Ada L, Dean CM, et al. Treadmill training is effective for ambulatory adults with stroke: a systematic review. J Physiother 2013;59:73-80.

93 Pollock A, Baer G, Campbell P, et al. Physical rehabilitation approaches for the recovery of function and mobility following stroke. Cochrane Database Syst Rev 2014:4:CD001920.

94 Pollock A, Gray C, Culham E, et al. Interventions for improving sit-to-stand ability following stroke. Cochrane Database Syst Rev 2014;5:CD007232.

95 Rao AK, Chou A, Bursley B, et al. Systematic review of the effects of exercise on activities of daily living in people with alzheimer's disease. Am J Occup Ther 2014:68:50-6.

96 Shen X, Wong-Yu ISK, Mak MKY. Effects of exercise on falls, balance, and gait ability in Parkinson's Disease. Neurorehabil Neural Repair 2016:30:512-27.

97 States RA, Pappas E, Salem Y. Overground physical therapy gait training for chronic stroke patients with mobility deficits. Cochrane Database Syst Rev 2009;3:CD006075.

98 Tomlinson CL, Patel S, Meek C, et al. Physiotherapy versus placebo or no intervention in Parkinson's disease. Cochrane Database Syst Rev 2013:9:CD002817.

99 Wallis JA, Taylor NF. Pre-operative interventions (non-surgical and nonpharmacological) for patients with hip or knee osteoarthritis awaiting joint replacement surgery--a systematic review and meta-analysis. Osteoarthritis Cartilage 2011:19:1381-95.

100 Chen YM, Zhu M, Zhang YX. Combined endurance-resistance training improves submaximal exercise capacity in elderly heart failure patients: a systematic review of randomized controlled trials. Int I Cardiol 2013;166:250-2.

101 Zhang WW, Speare S, Churilov L, et al. Stroke rehabilitation in China: a systematic review and meta-analysis. Int J Stroke 2014;9:494-502.

102 Rugbjerg M, lepsen UW, Jørgensen KJ, et al. Effectiveness of pulmonary rehabilitation in COPD with mild symptoms: a systematic review with meta-analyses. Int J Chron Obstruct Pulmon Dis 2015;10:791-801.

103 Wind J, Koelemay MJ. Exercise therapy and the additional effect of supervision on exercise therapy in patients with intermittent claudication. systematic review of randomised controlled trials. Eur J Vasc Endovasc Surg 2007:34:1-9.

104 Smidt N, de Vet HC, Bouter LM, et al. Effectiveness of exercise therapy: a bestevidence summary of systematic reviews. Aust J Physiother 2005:51:71-85.

105 Herring MP, Puetz TW, O'Connor PJ, et al. Effect of exercise training on depressive symptoms among patients with a chronic illness: a systematic review and metaanalysis of randomized controlled trials. Arch Intern Med 2012;172:101-11.

106 Pahor M, Guralnik JM, Ambrosius WT, et al. Effect of structured physical activity on prevention of Major mobility disability in older adults: the LIFE study randomized clinical trial. JAMA 2014;311:2387-96.

107 Carroll DD, Courtney-Long EA, Stevens AC, et al. Vital signs: disability and physical activity--United States, 2009-2012. MMWR Morb Mortal Wkly Rep 2014;63:407-13.

108 Balshem H, Helfand M, Schünemann HJ, et al. GRADE guidelines: 3. Rating the quality of evidence. J Clin Epidemiol 2011;64:401-6. 


\section{Correction: Exercise therapy for functional capacity in chronic diseases: an overview of meta-analyses of randomised controlled trials}

Pasanen T, Tolvanen S, Heinonen A, et al. Exercise therapy for functional capacity in chronic diseases: an overview of meta-analyses of randomised controlled trials. Br J Sports Med 2017;51:1459-65. doi:10.1136/bjsports-2016-097132.

Figure 4 legend should read: Effect of mixed exercise on functional capacity in chronic diseases.

C) Author(s) (or their employer(s)) 2020. No commercial re-use. See rights and permissions. Published by BMJ.

Br J Sports Med 2020;54:e4. doi:10.1136/bjsports-2016-097132corr1

(D) Check for updates 